\title{
Human-human haptic collaboration in cyclical Fitts' tasks*
}

\author{
Sommer Gentry and Eric Feron \\ Laboratory for Information and Decision Sciences \\ Massachusetts Institute of Technology \\ Cambridge, Massachusetts, 02139 \\ sommerg,feron@mit.edu
}

\author{
Roderick Murray-Smith \\ Dept. of Computing Science \\ University of Glasgow, Scotland \\ Hamilton Institute \\ Nat. Univ. of Ireland Maynooth \\ rod@dcs.gla.ac.uk
}

\begin{abstract}
Understanding how humans assist each other in haptic interaction teams could lead to improved robotic aids to solo human dextrous manipulation. Inspired by experiments reported in Reed et al. [1], which suggested two-person haptically interacting teams could achieve a lower movement time (MT) than individuals for discrete aiming movements of specified accuracy, we report that two-person teams (dyads) can also achieve lower MT for cyclical, continuous aiming movements. We propose a model, called endpoint compromise, for how the intended endpoints of both subjects' motion combine during haptic interaction; it predicts a ratio of $\sqrt{2}$ between slopes of MT fits for individuals and dyads. This slope ratio prediction is supported by our data.

Index Terms - haptic interaction, Fitts' law, human-human collaboration, rhythmic interaction, rhythm
\end{abstract}

\section{INTRODUCTION}

The vision of haptic collaboration is that a team of agents, interacting by touch, can work together to complete a task. A previous study demonstrated the superior performance of two-person haptically interacting teams in a one-dimensional discrete aiming task, also referred to here as a Fitts' task [1]. A discrete aiming task is to move a pointer to a stop inside a target as quickly as possible. In the present experiments, we expand this result to the cyclical aiming domain. Cyclical aiming does not require the pointer to come to rest in the target. Instead, the pointer should alternate between two targets as quickly as possible without overshooting or undershooting. The best strategy for low-difficulty cyclical aiming movements is to move in a smooth sinusoidal pattern. Because the pattern of muscle activation required should be substantially different between discrete and cyclical tasks, better performance for dyads in discrete tasks does not imply better performance for dyads in cyclical tasks.

Also, cyclical aiming requires timing coordination between the participants that is not required in discrete aiming. In a discrete aiming task, both people get a visual signal to end one aim and start the next. In cyclical aiming, there is no indication of when to finish aiming at one target and start aiming at the other, except the signals the subjects give to each other by pushing on the device that they share.

${ }^{*}$ S.G. is supported by a U.S. Department of Energy Computational Science Graduate Fellowship, and E.F. by a MURI grant number 0205G-CB222.

\section{BACKGROUND}

Fitts' law predicts that when humans perform minimumtime aiming tasks of distance $D$ with accuracy specified by the target's width $W$, the movement time (MT) achieved is

$$
M T=A \log _{2}\left(\frac{2 D}{W}\right)
$$

where the term $\log _{2}\left(\frac{2 D}{W}\right)$ is referred to as the index of difficulty (ID). Higher difficulty, more accurate movements require longer aiming time. Fitts' law is quite general, in that it has been shown to hold for single-joint and multiplejoint movements, on many scales, across differing pointing devices, and across differing feedback conditions [4], [5]. This trade-off between speed and accuracy in human motion is an ideal testing ground for experiments elucidating both the limits of human performance and the means by which human performance might be improved by cooperation with other individuals or with well-designed haptic devices. For instance, discrete Fitts' task performance can be improved by applying a cubic centering force to a joystick used to capture the target [6].

\section{A. Discrete versus cyclical}

Fitts first formulated his law for the cyclical case, in which a person is asked to aim successively at each of a pair of targets as rapidly as possible. In the alternative, discrete case, a person is asked to aim at and come to a stop within a given target.

Cyclical Fitts' tasks display an interesting phase shift [4]. Easy cyclical aiming has a harmonic or sinusoidal character, with the maximum acceleration corresponding to the extreme point of each movement. However, as the difficulty of the task increases, the cyclical task comes to resemble a discrete task in that each aim comes nearly to a full stop before the following aim begins. Discretization of the movements begins at index of difficulty between 4 and 5 . Guiard has demonstrated that, physically rather than informationally, sinusoidal motion gives cyclical aiming an MT advantage over discrete aiming in that sinusoidal motion permits storage and re-use of the kinetic energy a human has generated.

Note that harmonic motion has a distinct rhythm. Dyads especially might need to use rhythmic toeholds to enable 
cooperation, because if each partner started his next aim at a different time, he would have difficulty predicting the outcome of his force input on the device's position. This coordination issue does not arise in the discrete context because in discrete aiming, the start time is displayed to both individuals. Because the present experiments were intended to explore the space of cyclical, harmonic motion in dyads, the index of difficulty was deliberately kept low. IDs of 2.5 to 4.5 were tested in increments of 0.5 .

\section{B. Two-person Fitts' tasks}

Mottet et al. first explored two-person, or dyad, cooperative Fitts' tasks [7]. In their design, cooperation was purely informational: one person controlled the motion of the pointer, and another person controlled the motion of the target. Neither could feel the motion of the other; they could only watch the motion on a display. The investigators compared the performance of a dyad, with each subject moving either the target or the pointer, to the performance of a solo subject moving the target with one hand and the pointer with his other hand. Dyads performed the two-handed motion faster than individuals did. This finding was attributed to an informationprocessing cost to the individual of anti-phase coordination as the individual's two hands moved in opposite directions. However, dyads did not have any advantage over solo subjects who moved only the pointer, suggesting that information-only collaboration does not afford a true advantage.

Reed et al. had two subjects move a single pointer into a target region and stop the pointer's motion there [1]. Each of the subjects had a handle to turn the same physical crank, enabling haptic interaction. Dyads performed the movement an average of $140 \mathrm{~ms}$ faster than individuals could. Specifically, the limited data in this experiment suggested that the slope of the Fitts' law curve was the same for dyads and individuals. The haptic cooperation speedup was not conclusively explained, though the experimenters speculated that triphasic bursts of effort (agonist for takeoff, then antagonist and agonist for stopping [8]) could be more closely timed if different individuals took responsibility for different phases. Since the pattern of muscle activation for cyclical aiming tasks differs from the sketch above, we experimented with haptically coupled cyclical aiming tasks.

\section{Alternatives to Fitts' model}

Fitts' account of the relation

$$
M T=A+B \log _{2}\left(\frac{2 D}{W}\right)
$$

that he discovered in his data was based on Claude Shannon's information theory. The greater the information needed to specify exactly where to stop, Fitts reasoned, the longer the time a human would take to generate that movement. Fitts' law could not explain the variability of movement endpoints, and many other models have been proposed for the speedaccuracy tradeoff [9], [10].

Schmidt et al. found a very different shape for the speedaccuracy tradeoff when subjects were asked to tap alternately across a given distance at a given tempo [11]. Schmidt measured the velocity $\frac{D}{M T}$ and the standard deviation $\sigma$ of the movements' endpoints, and the data suggested that the latter was proportional to the former: $\sigma \propto \frac{D}{M T}$. That is, a movement's endpoint was approximately normally distributed, and its variance increased with the velocity of the movement. Interpreting the standard deviation of the movements' endpoints as the effective target width $W_{e}$, in Schmidt's task there is an approximate linear tradeoff

$$
M T=A+B\left(\frac{D}{W_{e}}\right)
$$

instead of a logarithmic one.

Our data were best explained by (3), using the actual target width, which yields the expression

$$
M T=A+B\left(\frac{D}{W}\right)
$$

\section{ADAPTING SCHMIDT'S LAW FOR DYADS}

Schmidt's law can make no predictions concerning dyadic performance without a model for how individual movement variabilities would combine during haptic interaction. The individuals are holding the same device, so are constrained to move at the same velocity and share the same movement endpoint. A simple and appealing model is endpoint compromise. With the endpoint compromise assumption, the individuals move to capture two independently selected movement endpoints, with the result that the pointer stops at the average of the endpoints. While we do not offer a physical justification for this assumption, we do hope to address that issue in future work. If $\sigma_{d}$ is the standard deviation of the average of two endpoints each with standard deviation $\sigma_{s}$, elementary calculations give

$$
\sigma_{d}=\frac{1}{\sqrt{2}} \sigma_{s}
$$

For fixed target width $W_{0}$, the individual or dyad is asked to adjust movement time until the probability that the movement endpoint lands outside the target width is less than 5\%, but not to move too slowly or aim more accurately than necessary. Lower variability should result in faster movement. Percentiles of the movement endpoint's (normal) distribution scale with standard deviation. Then, for a fixed target width $W_{0}$ the solo and dyad movement times $M T_{s}$ and $M T_{d}$ should approximately satisfy $M T_{d}=\frac{1}{\sqrt{2}} M T_{s}$. Endpoint compromise would predict that

$$
B_{d}=\frac{1}{\sqrt{2}} B_{s}
$$




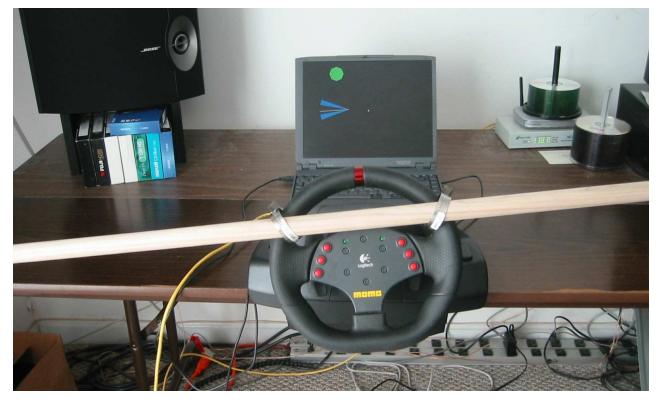

Fig. 1. Aiming device; one subject stood on either side of the wheel

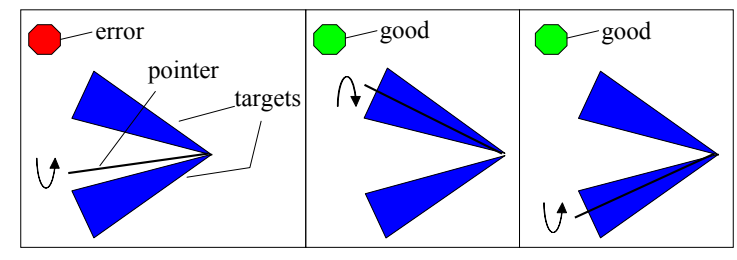

Fig. 2. Pointer should reverse direction while inside a target

where $B_{d}$ is the slope of (4) for $M T_{d}$, and the corresponding definition is made for $B_{s}$.

\section{ExPERIMENTAL PRotocol AND EQUipMEnT}

Five subjects participated in the experiment; two males and three females, all right-handed except one female was lefthanded. Although hand assignment does not affect Fitts' task performance [7], each subject used his dominant hand for all parts of the experiment. The subjects were not paid and had either no or little prior exposure to haptic devices.

Each subject performed a one-dimensional cyclical Fitts' task. They used a standard computer driving wheel fixed to a desk, with a $4 \mathrm{ft}$ long wooden dowel attached to create a lever, as pictured in Figure 1. The movements were approximately in a plane parallel to the wall behind the desk, so that the subjects aimed alternately up and down at the targets. The two targets were displayed on the computer's screen as sectors of a circle, with the current position marked as a pointer. The display always showed both stationary targets. An indicator in the upper left corner of the screen, colored green or red, corresponded to success or failure of the previous aim. A schematic of the task appears in Fig. 2.

Five levels of difficulty were tested. The two targets were fixed at $D=30^{\circ}$ apart, and the target widths used were $W=$ $10.6^{\circ}, 7.5^{\circ}, 5.3^{\circ}, 3.75^{\circ}$, and $2.65^{\circ}$, with index of difficulty (ID), defined as $\log _{2}\left(\frac{2 D}{W}\right)$, of $2.5,3.0,3.5,4.0$, and 4.5. All of the solo and dyad trials were conducted on the same day.

1) Solo trials: The solo experiment had two practice sessions, each consisting of five blocks of 60 aims for each of the five difficulty conditions. After each block of 60 aims, the error rate for that difficulty level was displayed. Participants were asked to try to keep their error rates below 5\%, but to minimize movement time within that constraint. After the practice sessions, data were collected for 120 aims between the stationary targets for each level of difficulty. During the solo experiment, the subjects all used the left side of the apparatus. Each solo trial lasted between twelve and fifteen minutes.

2) Dyad trials: Following the solo experiments, each participant was paired with every other participant in the experiment, for a total of ten dyads. Each dyad performed the cyclical aiming task together, with one person on either side of the rotating handle. Because the subjects were on opposite sides of the wheel, when one subject was moving the handle down, the other subject had to be moving the handle up. During the dyad tasks, each person had his own targets and pointer display, corresponding to the position of the handle on his side of the apparatus. There was no conflict between the information displayed to each subject. Subjects were assigned to the left side and to the right side of the apparatus in alternating order.

The dyad experiment had a single practice session consisting of five blocks of 60 aims for each of the five difficulty conditions. After each block, the error rate for that difficulty level was displayed. The instructions to minimize movement time subject to an error rate below 5\% were the same as in the solo experiment. After the practice sessions, data were collected for 120 aims between the stationary targets for each level of difficulty. Each dyad trial lasted between seven and ten minutes. Subjects did not complain of fatigue. Although subjects were invited to rest between any of the blocks of the experiments, only one individual and two dyads stopped for a break during the experiment.

\section{A. Data Analysis}

A computer timer with 10 milliseconds of precision measured the movement durations. The extreme point of each movement marked the end of one aim and the beginning of the next aim. If the extreme point of a movement was within the target sector, then the move was successful; otherwise, the movement was marked as an error. Within the 120 aim trials, the first 20 movements were discarded as warm-up. The remaining movement durations were averaged for each solo and dyad trial.

The angular position of the joystick was recorded as the program ran, but the capture rate was low, only 12 Hertz. Each movement, therefore, might have had as few as 3 or 4 recorded positions. The harmonicity of the overall movement, however, was clearly visible even with the low capture rate.

A linear least-squares fit between ID and MT, along with an $R^{2}$ value, was calculated separately for all five solo trials and all ten dyad trials to assess the validity of Fitts' law with respect to our data. Also, a linear least-squares fit between $(D / W)$ and MT was calculated separately for each trial to assess the validity of Schmidt's law with respect to our data. 
TABLE I

FIT OF MT (MS) TO FITTS' LAW AND SCHMIDT'S LAW

\begin{tabular}{rrr} 
Solo & $\log _{2}\left(\frac{2 D}{W}\right)$ & $\frac{D}{W}$ \\
\hline Slope & 428.2 & 102.1 \\
Intercept & -658.3 & 189.7 \\
$R^{2}$ & 0.9320 & 0.9813 \\
& & \\
Dyad & & \\
\hline Slope & 307.1 & 76.6 \\
Intercept & -496.4 & 90.9 \\
$R^{2}$ & 0.8109 & 0.9250
\end{tabular}

The slopes and intercepts for all solo and dyad trials were averaged. A summary of these results appears in Table I.

\section{RESUlts AND Discussion}

The linear least-squares fit to our movement time data was calculated for Fitts' $\log _{2}\left(\frac{2 D}{W}\right)$ and Schmidt's $\frac{D}{W}$. Table I shows that the fit to the term $\frac{D}{W}$ is much better, for both the solo and the dyad conditions. The term $\frac{D}{W}$ explains $98 \%$ of the data for individuals and $93 \%$ of the data for dyads. The results are most similar to those Schmidt obtained in experiments which were both time-constrained and spaceconstrained, in which people attempted to move an approximate distance at a given tempo. Table II contains average movement times, in milliseconds, for every subject and dyad.

Subjects could not make eye contact because the task demanded constant visual attention to the screen. No instructions were given regarding verbal negotiation, and a small amount of verbal negotiation did occur. All the conversations surrounded the tempo of the dyad's motion: either, "You're slowing me down," or "We can do this faster."

\section{A. Errors}

None of the individuals or dyads was able to achieve the requested $5 \%$ error rate in the difficult tasks. The number of errors and corresponding error rates for all trials are shown in Table III. As in nearly every Fitts' law experiment, higher error rates were observed for higher difficulty levels. The error rates for dyads and individuals are similar except at the two highest difficulty levels tested, where dyads committed more errors than individuals. This higher error rate might contaminate the finding of lower movement time for dyads, because the dyads did not move as accurately as the solo performers. Future experiments could financially reward accuracy and provide longer practice times to attempt to remove the accuracy gap between solo and dyad performers.

\section{B. Movement harmonicity}

The angle capture traces afford a qualitative evaluation of harmonicity. The profiles which were almost perfectly symmetric about the reversals of direction can be classified as harmonic, while the profiles with significantly more observations (shown as dots in the figures here) before the reversals than after the reversals can be classified as inharmonic.

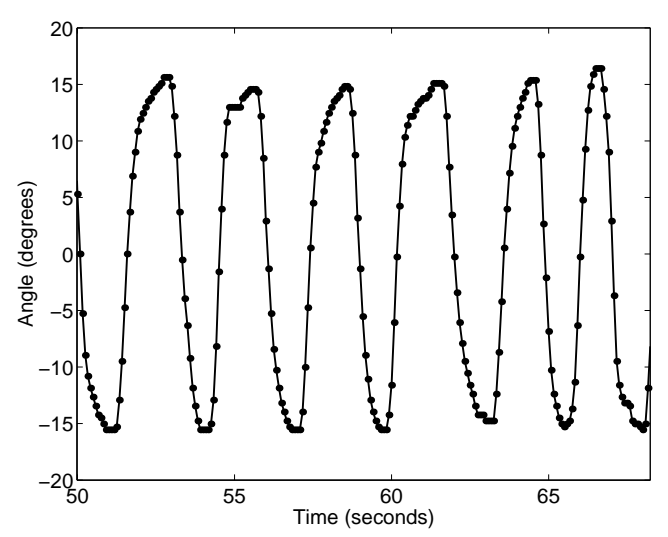

Fig. 3. Subject $B, I D=4.5$, inharmonic motion

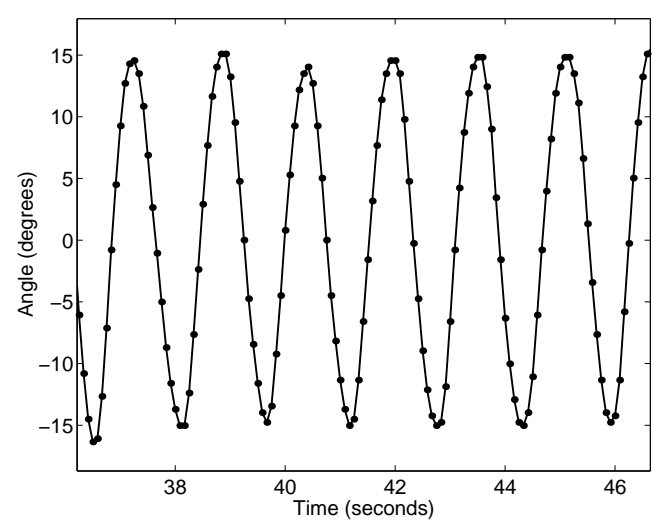

Fig. 4. Dyad D+B, ID=4.5, harmonic motion

The range of difficulties in the experiment included tasks difficult enough that harmonic motion was no longer possible for individuals. See Fig. 3 for a typical trace of an individual doing the most difficult task. Although individuals chose inharmonic, discretized motion for the ID $=4.5$ task, many dyads achieved harmonic motion for the most difficult task. For instance, Fig. 4 is an example of fairly smooth sinusoidal motion. Obviously these characterizations of the traces as harmonic or inharmonic are crude, and some traces seemed to contain a mixture of some harmonic reversals and some discretized reversals, as in Fig. 5.

All individual and dyad traces for the most difficult task, ID $=4.5$, were viewed and grouped into three categories: harmonic motion, mixed, and non-harmonic or discretized motion. Of the 5 individual traces, 3 were discretized and 2 were mixed. Of the 10 dyad traces, 5 were harmonic, 3 were mixed, and only 2 were discretized. The categorizations appeared to be unrelated to the error rate. Dyads were sometimes capable of harmonic motion in the hardest task, but no individuals were. This finding is consistent with the observation in Guiard [4] that movement harmonicity is related to high peak velocities. Movement harmonicity only indirectly decreases with the task difficulty because more 
TABLE II

AVERAGE MOVEMENT TIMES IN MILLISECONDS

\begin{tabular}{cccccccccccc} 
Solo & A & B & C & D & E & & & & & & Means \\
\hline Slope(ms/bit) & 107 & 89.2 & 129 & 77.6 & 108 & & & & & & 102.05 \\
Intercept (ms) & -56 & 495 & 138 & 101 & 271 & & & & & & 189.71 \\
$R^{2}$ & 0.99 & 0.96 & 0.99 & 0.99 & 0.98 & & & & & & 0.9813 \\
& & & & & & & & & & & \\
Dyad & $\mathrm{B}+\mathrm{A}$ & $\mathrm{A}+\mathrm{C}$ & $\mathrm{C}+\mathrm{D}$ & $\mathrm{D}+\mathrm{A}$ & $\mathrm{E}+\mathrm{D}$ & $\mathrm{A}+\mathrm{E}$ & $\mathrm{E}+\mathrm{C}$ & $\mathrm{C}+\mathrm{B}$ & $\mathrm{B}+\mathrm{E}$ & $\mathrm{D}+\mathrm{B}$ & Means \\
\hline Slope(ms/bit) & 89.5 & 88.5 & 71.8 & 83 & 65.1 & 82.5 & 101 & 67.4 & 72.3 & 45.1 & 76.6 \\
Intercept (ms) & 122 & 82.9 & 108 & 53.4 & 92.4 & 57.8 & -45 & 146 & 119 & 172 & 90.9 \\
$R^{2}$ & 0.98 & 0.92 & 0.86 & 0.95 & 0.96 & 0.99 & 0.93 & 0.87 & 0.9 & 0.89 & 0.92501
\end{tabular}

TABLE III

ERROR COUNTS: 6 ERRORS ARE 5\% OF 120 MOVEMENTS

\begin{tabular}{rrrrrrrr} 
Solo & $\frac{D}{W}$ & A & B & C & D & E & Means \\
\hline Errors & 2.81 & 3 & 0 & 0 & 2 & 0 & $0.83 \%$ \\
Errors & 4.00 & 7 & 1 & 4 & 1 & 4 & $2.83 \%$ \\
Errors & 5.66 & 4 & 3 & 10 & 1 & 12 & $5.00 \%$ \\
Errors & 8.00 & 14 & 7 & 11 & 10 & 11 & $8.83 \%$ \\
Errors & 11.32 & 9 & 7 & 12 & 8 & 18 & $9.00 \%$
\end{tabular}

\begin{tabular}{lrrrrrrrrrrrr} 
Dyad & $\frac{D}{W}$ & $\mathrm{~B}+\mathrm{A}$ & $\mathrm{A}+\mathrm{C}$ & $\mathrm{C}+\mathrm{D}$ & $\mathrm{D}+\mathrm{A}$ & $\mathrm{E}+\mathrm{D}$ & $\mathrm{A}+\mathrm{E}$ & $\mathrm{E}+\mathrm{C}$ & $\mathrm{C}+\mathrm{B}$ & $\mathrm{B}+\mathrm{E}$ & $\mathrm{D}+\mathrm{B}$ & Means \\
\hline Errors & 2.81 & 3 & 0 & 1 & 5 & 0 & 4 & 3 & 0 & 3 & 0 & $1.58 \%$ \\
Errors & 4.00 & 2 & 3 & 0 & 1 & 8 & 4 & 8 & 3 & 8 & 0 & $3.08 \%$ \\
Errors & 5.66 & 12 & 8 & 2 & 2 & 4 & 9 & 9 & 4 & 4 & 1 & $4.58 \%$ \\
Errors & 8.00 & 22 & 13 & 9 & 12 & 14 & 16 & 17 & 18 & 13 & 5 & $11.58 \%$ \\
Errors & 11.32 & 10 & 19 & 14 & 15 & 24 & 20 & 28 & 29 & 15 & 15 & $15.75 \%$
\end{tabular}

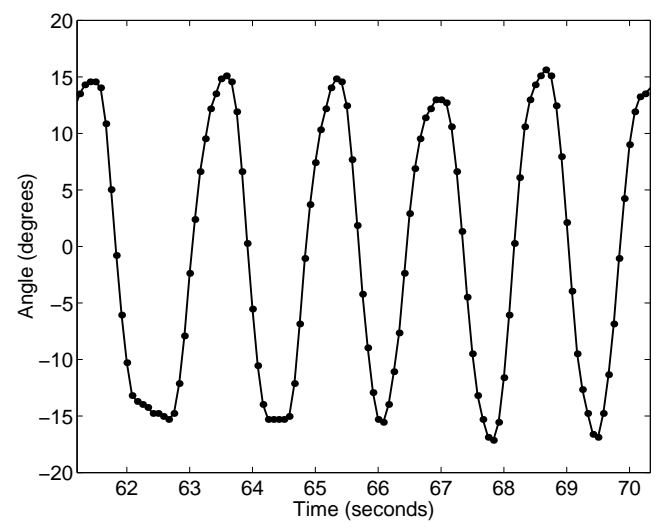

Fig. 5. Dyad $\mathrm{A}+\mathrm{B}, \mathrm{ID}=4$, mixed harmonic and discretized

difficult tasks must be done with lower peak velocities. The peak velocities observed in dyads were usually higher than those observed in solo trials.

\section{Line fits}

Dyads' intercepts are roughly 100 milliseconds lower than individuals' intercepts. The average difference between indi- vidual and dyad MTs was 99 with a $95 \%$ confidence interval of $(13,184)$. The $t$-distribution $(d f=18)$ of this difference was estimated using 20 observations, each a difference between an individual's and one of his dyads's intercept fits. The average MT's for every individual subject appear in Fig. 6.

Our slope hypothesis, as formulated in section III, was that the slope of the best fit to MT data for individuals would be larger than the slope of the best fit to MT data for dyads, by a factor of $\sqrt{2}$. Our data are consistent with this hypothesis. The observed ratio between the solo and dyad slopes was 1.37 , with a $95 \%$ confidence interval of $(1.22,1.51)$. The $t$-distribution $(d f=18)$ of this ratio was estimated using 20 observations, each a slope ratio between an individual's and one of his dyads' slope fits. This finding of different slopes is not consistent with the identical slopes reported for the discrete pointing task in [1].

\section{Practice effects and mechanical advantage}

Angle and movement time data were collected for all practice phases of the experiment. No significant learning effects were noted in movement harmonicity or error rates. For the solo experiment, movement times increased slightly from the first practice blocks to the final blocks. This is 


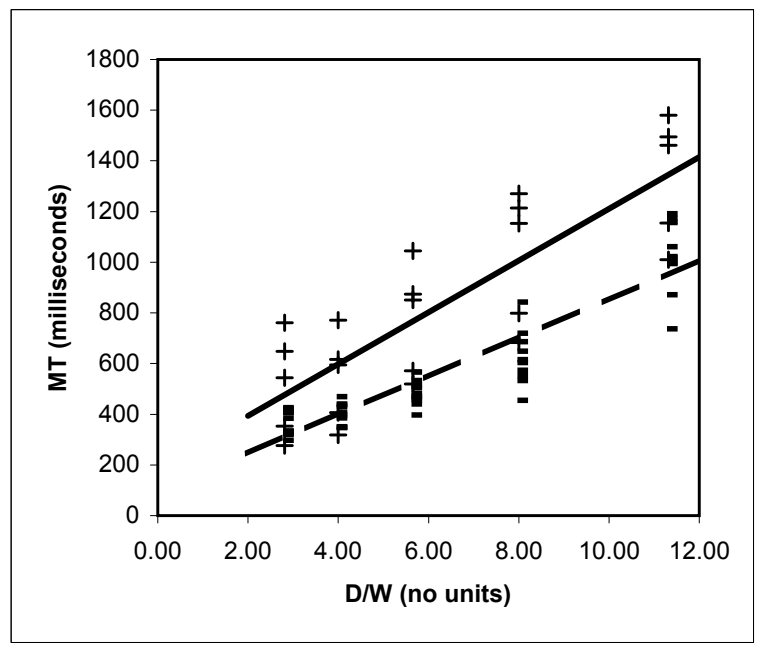

Fig. 6. Dispersion of average movement times for each subject (plus sign) and each dyad (dash), and averaged line fits for individuals (solid) and dyads (dashed).

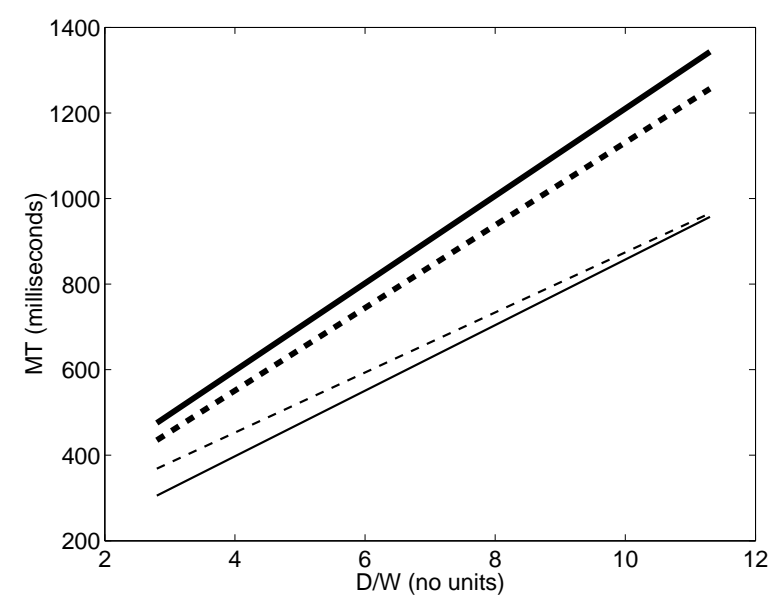

Fig. 7. With practice, the difference between solo and dyad performance becomes more pronounced. (Solo practice, thick dashed line; solo trials, thick solid line; dyad practice, thin dashed line, dyad trials, thin solid line)

because subjects needed to slow down from their initial speeds in order to keep error rates below $5 \%$.

For the dyad experiment, movement times decreased slightly from the first practice blocks to the final blocks. In both cases, the changes affected primarily the intercepts and not the slopes of the line fits. Since practice tended to make differences between dyad and solo performance larger, it is unlikely practice effects confounded the results. Fig. 7 shows plots of the average line fits to practice (dashed lines) and actual (solid lines) MTs.

The force required to move the handle did not fatigue the subjects. Still, it is difficult to rule out that the dyads performed better because two people could apply a larger force on the handle to reach a higher peak velocity than was possible for some of the individuals. In Figure 6, the lowest individual times were not achieved by the seemingly strongest individual subjects. A refined experimental apparatus might scale by a factor of two the force necessary to move the handle for the dyad case to resolve this question.

\section{CONCLUSION}

Dyads performed significantly better at a minimum-time cyclical aiming task than individuals. This finding extends the result on two-person improvements for discrete aiming tasks reported by Reed et. al [1]. The slope of the fit to Schmidt's law is lower for dyads than for individuals, and to explain this we propose the endpoint compromise hypothesis. Additionally, dyads sometimes maintained a sinusoidal motion at a higher difficulty level than any individual did in these experiments. Planned future work includes a physical justification for endpoint compromise, and experiments with expert dance partners to see whether their haptic collaboration skills transfer to this task.

\section{ACKNOWLEDGMENT}

The subjects, all members of the Information Control Engineering group at MIT, were generous with their time and helpful with their suggestions for the experimental interface. Zhi-hong Mao critically reviewed the manuscript.

\section{REFERENCES}

[1] K. Reed, M. Peshkin, J. E. Colgate, and J. Patton, "Initial studies in human-robot-human interaction: Fitts' law for two people," in Proceedings of IEEE International Conference on Robotics and Automation, pp. 2333-2338, 2004.

[2] A. M. Okamura, "Methods for haptic feedback in teleoperated robotassisted surgery," Industrial Robot, vol. 31, no. 6, pp. 499-508, 2004.

[3] D. Feygin, M. Keehner, and F. Tendick, "Haptic guidance: Experimental evaluation of a haptic training method for a perceptual motor skill," in IEEE Haptic Interfaces for Virtual Environment and Teleoperator Systems, pp. 40-47, March 2002.

[4] Y. Guiard, "Fitts' law in the discrete vs. cyclical paradigm," Human Movement Science, vol. 16, pp. 97-131, 1997.

[5] R. J. Jagacinski and J. M. Flach, Control Theory for Humans: Quantitative approaches to modeling performance. Mahwah, New Jersey: Lawrence Erlbaum Associates, 2003.

[6] D. Repperger, C. A. Phillips, and T. L. Chelletter, "A study on spatially induced 'virtual force' with an information theoretic investigation of human performance," IEEE Transactions on Systems, Man, and Cybernetics, vol. 25, pp. 1392-1404, October 1995.

[7] D. Mottet, Y. Guiard, T. Ferrand, and R. J. Bootsma, "Two-handed performance of a rhythmical fitts task by individuals and dyads," Journal of Experimental Psychology: Human Perception and Performance, vol. 27, no. 6, pp. 1275-1286, 2001.

[8] N. Lan and P. Crago, "Optimal control of antagonistic muscle stiffness during voluntary movements," Biological Cybernetics, vol. 71, no. 2, pp. 123-135, 1994.

[9] D. E. Meyer, S. Kornblum, R. A. Abrams, C. E. Wright, and J. K. Smith, "Speed-accuracy tradeoffs in aimed movements: Toward a theory of rapid voluntary action," in Attention and Performance XIII (M. Jeannerod, ed.), vol. 13, pp. 173-226, Erlbaum, 1990.

[10] D. Meyer, S. Kornblum, R. Abrams, C. Wright, and J. Smith, "Optimality in human motor performance: ideal control of rapid aimed movements," Psychological Review, vol. 95, no. 3, pp. 340-370, 1988.

[11] R. Schmidt, H. Zelaznik, B. Hawkins, J. Frank, and J. Q. Jr., "Motoroutput variability: A theory for the accuracy of rapid motor acts," Psychological Review, vol. 86, pp. 415-451, 1979. 